\title{
Proceedings of the Meeting of The British Neuropsychiatry Association, Bristol, 30 June 1989
}

FUNCTIONS OF NEURONAL NETWORKS IN THE HIPPOCAMPUS AND CEREBRAL CORTEX IN MEMORY

ET Rolls. University of Oxford

To clarify how the hippocampus is involved in memory, a neuronal network model of the hippocampus and its return projections to the neocortex based on its connections and synaptic modifiability is proposed. The recurrent collateral system of the CA3 pyramidal cells enables any one CA3 cell to connect to any other CA3 cell with a contact probability of approximately $5 \%$, using Hebb-modifiable synapses. It is therefore proposed that the CA3 cells form an autoassociation matrix memory, which provides a basis for episodic memory, and have the properties of completion of partial input stimuli, generalisation, and gracefu degradation. The dentate granule cell stage which precedes the CA3 pyramidal stage may operate as a competitive neuronal network which with the low contact probability of the mossy fibres from the dentate to the CA3 cells operates to produce a sparse and non-redundant representation of information required for the CA3 autoassociation stage to operate correctly. It is suggested that the CA1 stage which follows the CA3 stage acts by competitive learning to produce an efficiently encoded signal that is returned by the backprojections to the neocortex to guide information storage there according to the (often multimodal) associations detected by the hippocampus. The widespread backprojections end in layer 1 of the neocortex, and it is suggested that the sites of storage in the neocortex are on those cortical pyramidal cells at which there is conjunctive forward and backprojected information, so that Hebbian learning could operate to modify the synapses from active forward and backprojected axons onto activated cortical pyramidal cells. During recall, the original pattern of cortical activity produced by the forward projections would be elicited by the backprojections operating through the modified synapses. Some neurophysiological predictions of the theory have been tested.

1 Rolls ET. In: The representation and storage of information in neural networks in the primate cerebral cortex and hippocampus. primate cerebral cortex and hippocampus.
Durbin $\mathrm{R}$, Miall $\mathrm{C}$, Mitchinson $\mathrm{G}$, eds. The Durbin R, Miall C, Mitchinson G, eds. The computing neuron.

THE RELATION BETWEEN ANTEROGRADE AND RETROGRADE AMNESIA IN ALCOHOLOKORSAKOFF'S SYNDROME

AJ Parkin. University of Sussex

Patients with Korsakoff's syndrome exhibit both anterograde and retrograde amnesia. Anterograde deficits are manifest in patients inability to recall or recognise new verba and non-verbal information. Retrograde amnesia is evident from patients' poor recollection of premorbid experiences and, more formally, by their failure on tests such as "famous faces". Here the patient is required to identify people who have become famous in different decades. Korsakoffs show poorer overall memory and a temporal gradient where performance gradually deteriorates as more recent aspects of remote memory are tested.

The anterograde deficit in Korsakoff's syndrome has been interpreted as a failure to encode contextual information. The study examined whether retrograde amnesia might reflect the same contextual deficit-the idea being that a single contextual processing system underlies both the formation of new memories and the organisation and retrieval of existing memories.

This contextual deficit theory was examined by comparing the naming of famous faces with minimal contextual cues compared with a context condition in which relevant contextual information about each face was also present. Control subjects performed better in the context condition and showed no evidence of a temporal gradient. Those with Korsakoff's syndrome showed a temporal gradient and a declining influence of context in more recent remote memory.

The data are inconsistent with a contextual deficit theory of Korsakoff retrograde amnesia since this would have predicted greatest contextual enhancement for more recent periods of premorbid memory. Two alternative explanations of Korsakoff retrograde deficits remain. First, the deficit could reflect an incipient and worsening anterograde amnesia in the premorbid period due to the effects of chronic alcoholism. Some support for this view comes from studies which have shown a correlation between anterograde deficits and retrieval from more recent periods of remote memory. However, the fact that temporal gradients can be observed in patients without a history of chronic alcoholism suggests that other factors, namely storage deficits, must contribute to the pattern of deficits seen in Korsakoff's syndrome.

THE FOREBRAIN CHOLINERGIC SYSTEM AND MEMORY IN THE RAT: EFFECTS OF EXCITOTOXIC LESIONS, CHRONIC ALCOHOL AND FOETAL BRAIN TISSUE IMPLANTS

JA Gray, H Hodges, Y Allen, J Sinden, P Lantos. Institute of Psychiatry, London

Ibotenic acid lesions to nucleus basalis, medial septal and diagonal band brain regions induced marked deficits in spatial and associative radial maze tasks in rats. Lesioned rats were also more sensitive than controls to the deleterious effects of cholinergic antagonists (scopolamine and mecamylamine) on performance and, unlike controls, showed improvements after cholinergic agonists (arecoline and nicotine), possibly through the occurrence of denervation supersensitivity.

After cholinergic-rich (basal forebrain) or -poor (hippocampal) foetal cell suspension transplants into either the terminal (cortex, and/or hippocampus) or source (lesioned) regions of cholinergic forebrain projections, only the three cholinergic-rich terminal transplant groups showed improvements during weekly radial maze testing. These were significant from seven to nine weeks after transplantation and comparable in all groups, irrespective of transplant site. All lesioned and transplant groups continued to show greater sensitivity to cholinergic drugs than controls. This was most marked in the behaviourally effective transplant groups, where low doses of nicotine impaired performance, akin to a high dose action. Cortical and hippocampal choline acetyltransferase activity showed site-related increases in the cholinergic-rich terminal transplant groups, though activity was significantly reduced in lesioned and all other transplant groups. Hence cholinergic-rich terminal but not distal transplants promoted functional recovery, consistent with a local paracrine mechanism of action. Despite behavioural improvement, supersensitive response to cholinergic drugs was enhanced, suggesting an additive interaction between host and transplant receptors. Very similar results were obtained in animals in which comparable cognitive deficits were induced by a 28 week consumption of alcohol via the drinking water; this regime caused damage to the forebrain cholinergic system similar to that caused by ibotenic acid lesions.

AMNESIA: LESION LOCATION AND FUNCTIONAL DEFICIT - WHAT IS THE LINK?

Andrew Mayes. University of Manchester

Knowledge about the location of the brain lesions that cause amnesia should constrain the range of acceptable hypotheses about the functional deficit(s) that underlies this syndrome. There are three main views about the critical brain damage. The first view is that hippocampal or hippocampal circuit damage alone causes amnesia. The second view is that damage to this system must occur conjointly with amygdalar circuit damage if severe, permanent amnesia is to result. The third view is that the amnesic effects of hippocampal system damage are exacerbated by damage to some or all of the polysensory regions that project to it and to certain medial diencephalic sites. Curren evidence inconclusively favours the third view over the second and is inconsistent with the first. Our knowledge of hippocampal anatomy and physiology suggests that it may tag neocortically processed and stored sensory information with contextual markers. This is compatible with the contextmemory deficit hypothesis (CMDH) according to which amnesia is caused by a selective deficit in direct memory for information about backgroud spatiotemporal context and mode of presentation. The $\mathrm{CMDH}$ predicts that amnesics will be disproportionately bad at memory for various kinds of context relative to target information and that the extent of this disproportionate deficit will correlate with severity of amnesia. These predictions were tested in a group of 17 amnesics of mixed aetiology with a) crossmodal memory, b) memory for mode of word presentation, and c) spatial memory. All three kinds of memory were disproportionately impaired in the amnesics regardless of their aetiology. The degree to which cross-modal memory was disproportionately 
impaired did not correlate with intelligence, target memory or frontal signs and it was argued that this deficit was caused by amygdalar circuit damage, which had little effect on amnesia. Spatial and mode memory were unrelated in normal subjects and amnesic patients and each correlated significantly with amnesic severity as the $\mathrm{CMDH}$ predicts.

\section{SELECTIVE MEMORY DEFICITS IN PARKINSON'S} DISEASE

HJ Sagar. Royal Hallamshire Hospital, Sheffield

Parkinson's disease (PD) provides a potential clinical model to evaluate the role of neurochemical processes, especially dopamine, in the function of normal memory. Interpretation of the aetiology of memory impairment in PD is, however, complicated by the coexistence of depression, motor delays, side effects of drugs, and cortical pathology, such as Alzheimer's disease (AD). Accordingly, a series of experiments have been carried out to determine a) the nature and specificity of memory impairment in $P D, b$ ) the relevance of confounding clinical factors, and c) the effect of dopamine replacement. The studies included a comparison of $P D$ and $A D$ patients and evaluation of newly diagnosed, non-demented PD patients before and after treatment. The results showed that memory impairment in PD was 1) present at diagnosis, 2) involved short-term or working memory more than long-term memory, 3) affected explicit but not implicit memory, 4) was qualitatively different from $A D, 5$ ) was independent of depression, dementia, drug treatment and motor artefacts, and 6) did not respond to dopamine replacement. The results suggest that the early retention of information in memory is sensitive to specific non-dopaminergic pathology of PD.

\section{BRAIN INJURY: MEMORY DISORDERS THE} THEORIES DON'T EXPLAIN

Peter Eames. Burden Neurological Hospital, Bristol

It is impressive that most disorders of memory and attention in those with brain injuries fit quite well with current views of the nature of memory. It is therefore even more impressive to find individuals with abnormalities of memory that do not fit, yet may advance understanding of memory mechanisms. Three examples were discussed.

Three patients encountered over ten years believed they had very poor memory, but if pressed for answers could usually be accurate but not recognise a response as being correct. This "knowing without knowing that you know" might result from abnormality of the "familiarity" centre demonstrated in animals in the temporal cortex. Each patient had suffered very severe diffuse brain injury, but had severest deficits in recognition memory.

Five patients described as having poor memory and "confusion" made good steady recovery of performance on memory testing. All had normal Rivermead Behavioural Memory scores by six months. Their disorder consisted of talking and behaving as though in another time, place and role. Initially this was persistent, but gradually became episodic, and within two years the episodes had petered out, eventually being seen only with excitement or distress. Clearly these were reduplicative disorders, exacer- bated by arousal. Since the phenomena shared the affective tone of certainty seen in deja-vu, they might also result from malfunction of "familiarity" mechanisms.

The third group had suffered brain insults of the most diffuse kind. Formal or informal testing, or very direct questioning, suggested profound amnesia, yet behavioural observation showed efficient use of recently acquired information. The severest showed little tendency to recover, but those without persisting neurological deficit improved over several years. Apart from the obvious resemblance to hysteria, this disorder has much in common with ideomotor dyspraxia, which these patients showed also at a behavioural level: the one response that cannot be made is the response explicitly required.

REDUPLICATIVE PARAMNESIA ASSOCIATED WITH A LEFT OCCIPITO-PARIETAL HAEMATOMA RA Metcalfe, D Neary, B Northern. Manchester Royal Infirmary

A 64 year old right handed man was admitted to hospital with a history of sudden loss of vision in both visual fields but sparing central vision. The visual loss resolved partially leaving a dense right homonymous hemianopia. Minor word finding difficulties, dyslexia and amnesia were evident initially but rapidly resolved. A CT scan demonstrated a left occipito-parietal haematoma.

It was only some weeks later that he reported a curious conviction which he had entertained whilst in hospital, namely that he was in London. He was able to maintain this conviction despite the evidence of his senses and felt that the hospital was Manchester Royal Infirmary but in London. This conviction gradually faded over the course of a few weeks on his return home.

This phenomenon, reduplicative paramnesia was first properly described by Benson in 1976. Most reported cases occur in the context of focal right hemisphere disease superimposed upon more diffuse damage usually related to head injury or alcoholism. The case presented is unusual in that the major disorder was in the posterior dominant hemisphere and there was no evidence of more diffuse disease, though the initial pattern of visual loss and the amnesia does suggest some bilateral cerebral disturbance.

COGNITIVE FUNCTION AFTER SELECTIVE AMYGDALO-HIPPOCAMPECTOMY FOR INTRACTABLE TEMPORAL LOBE EPILEPSY Charles E Poikey. Maudsley Hospital, London

Nineteen patients had selective amygdalohippocampectomy. In fourteen patients the pathology was mesial temporal sclerosis, in three patients some other pathology, and in two patients non-specific changes were found. There were sixteen patients in whom there was more than six months follow-up and five of these were free from fits and a further seven were improved.

A standard battery of neuropsychological tests were given pre-operatively and between four and six weeks post-operatively. Thirteen of these patients had also had carotid amytal tests to test speech dominance and memory function.

In general the effects of the operation were slight and variable. No significant changes were seen as a result of operation and only a few subtests were changed significantly in relation to the side of operation. There was a significant deterioration in the Benton test regardless of the pre-operative memory state of the operated hemisphere, but in the unoperated hemisphere only if the preoperative memory score in that hemisphere was moderate but not if it was poor. The occurrence of febrile convulsions before operation had no interaction with surgery but the age of insult affected the performance on the Rey delayed memory recall, in that if this was six years or greater the performance deteriorated whereas if it was five years or less performance was improved.

These preliminary results are difficult to interpret because of the small sample size and because they are possibly not appropriate tests of amygdala or hippocampal functions. Comparison with the results of "en bloc" resections is clearly important and is in progress.

RETROGRADE AND ANTEROGRADE AMNESIA IN RESEARCH AND PRACTICE

MD Kopelman. St Thomas's Hospital, London

In recent years neuropsychologists have made considerable advances in increasing our understanding of the pattern of memory deficits in amnesic and dementing disorders. One of the main findings to emerge is that there tends to be only a relatively low correlation between impairment on tests of retrograde and anterograde memory. Individual patients have been reported who show a disproportionate impairment on one or other of these components of organic amnesia. Even within these components, claims have been made for disproportionate losses of certain aspects of knowledge, for example De Renzi et al in Italy reported a patient who could remember personal facts about his past, but not more public information. A study was reported, which was conducted at the Institute of Psychiatry, looking at these issues in detail in a sizeable group of Korsakoff and Alzheimer patients. From the findings, it was argued that lesions within the limbic-diencephalic circuits give rise to an anterograde amnesia with a relatively brief (temporally-limited) retrograde amnesia, whereas it is the combination of limbic-diencephalic pathology and the presence of frontal dysfunction which appears to produce a temporally-extensive retrograde amnesia.

Such observations have implication for our understanding of neurochemical systems and their role in memory. It was suggested that cholinergic depletion gives rise to an anterograde amnesia, but not a retrograde amnesia, and this has implications for pharmacological studies. Secondly, these kinds of observation can be useful in the assessment of individual clinical patients, and example cases were reported.

\section{KORSAKOFF'S SYNDROME IN A BOY AGED} 10 YEARS

M Prendergast. Birmingham Children's Hospital

Mental state changes were described in a boy aged 10 with tuberculous meningitis. $\mathrm{He}$ emerged from coma, into a fluctuating confusional state with verbigeration. Lucid intervals revealed a Korsakoff deficit in acquisition of new memories. He failed to learn the names of nurses or other children on 
the ward, or which bed was his. He insisted he was nine years old and it was impossible to persuade him he had become 10 whilst he was ill. Memory for events before his illness was unimpaired and reading and writing were unaffected. The confusional state cleared and memory for new material gradually improved. Confabulation replaced catastrophic reactions when he was questioned. Confabulation content was fantastic, but compatible with his previous remembered experiences. Inability to remember the names of new nurses was the earliest sign of relapse traced to poor drug compliance and the appearance of new tuberculomata.

Associated behaviour problems were demonstrated on videotape and their management and significance were discussed.

\section{A 19th century description of paroxysmal kinesiogenic choreoathetosis}

Paroxysmal choreoathetosis was described in familial form by Mount and Reack in 1940, ${ }^{1}$ while the kinesinogenic variant of the disease was reported by Kertesz in $1967 .^{2}$ An exhaustive review of the clinical spectrum of paroxysmal motor dyskinesias was made by Lance in $1977 .^{3}$ Current literature agrees that probably the oldest description of paroxysmal choreoathetosis was made by Gower in 1901 as "reflex epilepsy".4

The 19th century physiologist Angelo Mosso (1846-1910) is well known for his studies on muscular fatigue and the mechanical apparatus to measure it (Mosso's ergometer). In 1884, in a book entitled "Fear" dealing with the different motor and autonomic expressions of fear in humans, Mosso wrote "A man in Germany told me some very strange things about his excitability. Amongst others, he said he had to give up dancing because his legs would give way under him at the slightest emotion. Everything made him feel topsyturvy, just offering his arm to a lady to lead her into dinner, or crossing a room full of distinguished people, because the mere idea of being watched would make him shake and stagger about like a drunkard. This disease is known to doctors by the name agoraphobia which means fear of being in public".

The description of these motor abnormalities deserves some comment. In the original Italian "mancar sotto le gambe" (legs giving way) describes a condition of a mixed pattern of inhibition of the muscle tone with involuntary movements, and in a similar way, the terms "tremare e traballare" (to shake and stagger) can be correctly translated in to modern medical language by "choreoathetotic movements".

In a subsequent chapter of the same book, ${ }^{5}$ Mosso discussed the relations between fear and the chorea and ballismus, while in this description no permanent alteration in motricity were noted, implying that the patient's dyskinesias were intermittent and he was able to describe the critical abnormal motor disorder.
Mosso concluded the description by making a diagnosis of agoraphobia, but it is reasonable to consider paroxysmal kinesiogenic choreoathetosis (PKC) an alternative, more probable diagnosis. In fact, the motor disturbances of Mosso's patient showed some peculiar aspects. The first one is that all the hyperkinesiae were triggered by a motor activity, such as, dancing or crossing a room, sometimes performed in a balletic manner (for example, offering support to a partner). The second one is represented by the fact that all abnormal movements were accompanied by embarrassment ("the slightest emotion", being watched by "distinguished people"). Thus Mosso's patient suffered from a disease which was paroxysmal, kinesiogenic, triggered by anxious situations, and expressed itself by conscious choreoathetotic movements, sometimes associated with a lowered antigravitional tone. ${ }^{23}$

It seems possible that, like other motor disorders, ${ }^{67}$ Mosso's description preceded the clinical definition of paroxysmal kinesogenic choreoathetosis.

P MARTINEILI A S GABELLINI Institute of Neurology, Institute of Neurology,
University of Bologna, Bologna, Italy

1 Mount LA, Reack S. Familial paroxysmal choreathetosis in a patient with familial ataxia. Arch Neurol 1940;44:841-6.

1 Kertesz A. Paroxysmal kinesigenic choreathetosis. One entity within the paroxysmal choreathetosis: description of 10 cases including 1 autopsied. Neurology 1967;17:

$680-90$.
Lance JW. Familial paroxysmal dystonic choreathetosis and its differentiation from related syndromes. Ann Neurol its differentiation

4 Gowers WR. Epilepsy and other chronic convulsive diseases: their cause, symptoms and treatment. London: Churchill,
thersich

5 Mosso A. La Paura. Milano: Fratelli Treves Editori. 1884;8:181.

6 Calne DB, Dubini A, Stern G. Did Leonardo describe Parkinson's disease? N Engl J Med 1989;9:594.

7 Stern G. Did Parkinsonism occur before 1817? J Neurol Neurosurg Psychiatry 1989 (Special supplement); 11-12. 